\title{
El viejo problema del analfabetismo sigue en pie: el escenario educativo en la provincia de Buenos Aires durante la década del '40
}

\section{The old problem of illiteracy remains: the educational scenario in the province of Buenos Aires during the 40 s.}

Eva mara Petitti ${ }^{1}$

\section{RESUMEN}

En este trabajo nos proponemos indagar en la problemática del analfabetismo en la provincia de Buenos Aires durante la década del `40, centrándonos especialmente en vísperas del gobierno de Domingo Mercante (1946-1952) y en los primeros años de su gestión. Para ello tomamos dos ejes. El primero tiene que ver con el plano de las percepciones, es decir, con los diagnósticos realizados por los funcionarios políticos antes y luego del ascenso de Domingo Mercante como gobernador. El segundo esta relacionado con la heterogeneidad del escenario educativo de la provincia de Buenos Aires durante esa década. Enmarcándonos en los trabajos que analizan el peronismo desde una perspectiva regional, elegimos el caso de la provincia de Buenos Aires, no sólo por su gravitación en la vida política argentina sino por ser la provincia con mayor población en edad escolar e inmigración interna

Palabras Clave: Analfabetismo. Sistema educativo. Provincia de Buenos Aires. Domingo mercante.

\section{ABSTRACT}

In this study we will investigate the problem of illiteracy in the province of Buenos Aires during the decade of 40. We focus especially on the eve of the Domingo Mercante government (1946-1952) and in the early years of his administration. To accomplish this task, will we analyze two issues. First, the level of perceptions, namely, the diagnoses made by political officials before and after the promotion of Domingo Mercante as governor. Second, the heterogeneity of the educational scene in the province of Buenos Aires during the decade. Anchored in existing studies analyzing Peronism from a regional perspective, we chose the case of the province of Buenos Aires, not only for their influence on political life in Argentina but also, because is the province has the highest school age population and internal migration.

Keywords: Illiteracy. Education system. Province of Buenos Aires. Domingo mercante.

\footnotetext{
${ }^{1}$ Profesora y Licenciada en Historia por la UNMdP.
} 


\section{Introducción}

Si el peronismo ha sido uno de los temas mas indagados en la historiografía de América Latina, en los últimos años han cobrado importancia los estudios sobre sus variedades regionales y ha comenzado a seguirse esta línea en el ámbito de la esfera educativa. ${ }^{2}$ Enmarcándonos en estas nuevas corrientes centramos nuestro foco en la provincia de Buenos Aires como un escenario adecuado para nuestra investigación. En primer lugar cabe mencionar su relevancia como espacio decisivo para la conquista y el mantenimiento del poder político. Por ejemplo, en 1916 cuando Hipólito Yrigoyen, asumió a la presidencia, decretó la intervención de la provincia con el objetivo de quebrar un baluarte conservador. Asimismo, durante la llamada década "infame" la provincia de Buenos Aires constituyó una base de apoyo a la vez que un desafío a la coalición gobernante que controlaría la vida política del país hasta 1943. (WALTER, 1987). ${ }^{3}$

Pero además es un observatorio relevante tanto por su peso demográfico como económico. Entre 1895 y 1947, fruto de la afluencia inmigratoria y el crecimiento vegetativo, la población de la provincia de Buenos Aires se cuadriplicó. Los efectos de la crisis de 1930 y la Segunda Guerra Mundial significaron una modificación sustancial en el perfil productivo y un incremento de la inmigración interna que contribuyó a acelerar el proceso de urbanización que ya había sido importante en el período anterior. Cuando el peronismo asumió el gobierno, la población de la provincia, cuya extensión abarca el 10\% del territorio nacional, representaba más del $25 \%$ de los habitantes del país, de los cuales casi tres cuartas partes vivían en áreas urbanas. Asimismo reunía más del 70\% del valor de la producción industrial y de los obreros del país que mayormente se concentraban en el conurbano bonaerense. ${ }^{4}$

Si bien a partir de estas características, puede ser vista como un caso excepcional en relación a las demás, la provincia de Buenos Aires ocupó un lugar central en la definición de las políticas educativas nacionales. Incluso, como sostiene Pablo Pineau, fue el lugar donde se ensayaron, aunque no en forma premeditada, "las distintas propuestas educativas que luego implementaron o intentaron implementar, a nivel nacional." (1997: 19). De hecho, en esta provincia no solo se halla contenida la matrícula escolar más importante del país, ${ }^{5}$ sino también

\footnotetext{
${ }^{2}$ Entre otros estudios véase Macor y Tcach (2003), Panella (2005), Melón Pirro y Quiroga (2006). En lo que respecta a la educación desde ámbitos regionales véase Puiggrós (1997) y Hirschegger (2010).

${ }^{3}$ El golpe de estado de 1930 que derrocó al gobierno de Yrigoyen, inauguró la década "infame" caracterizada por una participación política restringida de fraude electoral que culminó con el golpe nacionalista de 1943.

${ }^{4}$ Walter (1987), Germani (1987).

${ }^{5}$ A comienzo de los años '40, la matrícula de la provincia de Buenos Aires representaba más de un $22 \%$ del total. El Censo Escolar de 1943, señala para el total del país una matrícula de 1.700 .327 alumnos en edad escolar (entre 6 y 13 años) y para la Buenos Aires de 380.651. Era seguida en orden de importancia numérica por Santa Fe (187.483) y Córdoba (174.177). (IV Censo Escolar de la Nación 1943).
} 
los efectos de la educación primaria en su territorio trascienden los alcances geográficos, habida cuenta del peso de las migraciones internas e internacionales. ${ }^{6}$

En un contexto en que los estudios sobre el peronismo están siendo objeto de revisiones que ponen el acento en las continuidades, ${ }^{7}$ el análisis de la educación primaria en la provincia de Buenos Aires no escapa a esta tendencia. ${ }^{8}$ Consideramos que un punto de partida para poner a prueba este supuesto, es preguntarnos acerca de cuál era el escenario educativo en la provincia de Buenos Aires en vísperas del gobierno de Mercante. Incluso aquellos autores que se han centrado en la escuela primaria como herramienta de adoctrinamiento, han considerado la relevancia de la acción educativa durante el peronismo (PLOTKIN, 2004; REIN, 1998). Sin embargo, si bien se ha destacado que en la década del '40 la antigua problemática del analfabetismo continuaba siendo una fuente de preocupaciones (REIN, 1998; SOMOZA RODRÍGUEZ, 2006), se ha señalado que el escenario educativo que recibió el peronismo era favorable en relación a otras áreas (TORRE; PASTORIZA, 2002).

El Censo General de la Nación indica que para 1947 el porcentaje de población mayor de 14 años que no sabía leer y escribir, era de un $13.6 \%$ en Argentina y de un $9.8 \%$ en la provincia de Buenos Aires (SECRETARIA TÉCNICA. IV Censo General de la Nación, 1947, t. I, v.1). Estos datos generales acompañados de una mirada retrospectiva de los censos anteriores ${ }^{9}$, parecen demostrar que en vísperas del peronismo el proyecto alfabetizador sarmientino había dado resultado. Sin embargo, ocultan dos cuestiones. Por un lado, la preocupación que en la década del '40 persistía respecto al analfabetismo, tal como expresaba claramente en 1942 el gobernador Rodolfo Moreno ante la Cámara de Diputados "el viejo problema del analfabetismo sigue en pie. Mas de 50.000 niños no concurren a escuela alguna y si a ello se suma la deserción de los que abandonan el aula sin cumplir la instrucción mínima que establece la ley_ en estado de semianalfabetismo_ la cifra a la que llegamos es desconsoladora" (Diario de Sesiones de la Cámara de Diputados, 1942, p. 17-18). Por otro lado la diversidad educativa existente, en la cual los alumnos de la provincia en tanto actores sociales, se encontraban distribuidos a lo largo de un escenario heterogéneo.

Antes de profundizar en cada uno de estas cuestiones, realizamos una aproximación tanto en lo que respecta a la normativa vigente como a los establecimientos educativos creados hasta ese entonces, con el objetivo de contextualizar el sistema educativo bonaerense durante la década del '40. En el segundo apartado nos proponemos analizar el escenario educativo en el plano de las

\footnotetext{
Pastoriza y Torre señalan que "Los 8.000 provincianos que recibía anualmente hasta 1936 pasaron a un promedio de 70.000 entre 1937 y 1943 y ascendieron hasta 117.000 entre 1944 y 1947. En total, sumaron un millón de nuevos residentes a Buenos Aires y si cinturón urbano [ ]". (TORRE; PASTORIZA, 2002, p. 262).

${ }^{7}$ Dos estudios profundos acerca de las transformaciones en la historiografía sobre el peronismo en la última década son los realizados por Acha y Quiroga (2009) y Palacio (2010).

${ }^{8}$ El trabajo de Pinkasz y Pitelli (1997) sobre las reformas educativas en la provincia de Buenos Aires, señalan varios aspectos de continuidad entre el gobierno de Manuel Fresco y Domingo Mercante.

${ }^{9}$ Estas proporciones en 1914, eran del 35.9\% y el 31.6\% respectivamente. Cabe aclarar que en 1914 se consideró analfabeta a toda persona mayor de siete años de edad (SECRETARIA TÉCNICA, IV Censo General de la Nación, 1947).
} 
percepciones, respondiendo los siguientes interrogantes: ¿cuáles fueron los diagnósticos, respecto a la persistencia de esta problemática que elaboraron los dirigentes políticos al asumir el gobierno Domingo Mercante? y ¿en qué se diferenciaron de aquellos realizados durante las gestiones anteriores? Finalmente tomando variables desagregadas tales como el espacio urbano y rural, los diferentes distritos, género y edad, nos preguntamos ¿cuál era la situación de la educación primaria considerando las diferentes regiones de la provincia de Buenos Aires durante la década del ' 40 ?

\section{Una aproximación a la educación primaria en la provincia de Buenos Aires.}

Al asumir el gobierno Domingo Mercante en 1946, continuaba en vigencia la Ley de Educación Común número 988 sancionada en 1875, que establecía la conducción del sistema educativo provincial en manos, por un lado de un Consejo General de Educación, integrado por un Director General nombrado por el Poder Ejecutivo con acuerdo del Senado, y ocho consejeros seleccionados por el Poder Ejecutivo con acuerdo de la Cámara de Diputados. Por otra parte los gobiernos locales estaban a cargo de los Consejos Escolares de Distrito con autoridades designadas por elección directa. ${ }^{10} \mathrm{El}$ primer reglamento escolar sancionado en 1876 dividía a las escuelas primarias en elementales (hasta tercer grado) y complementarias (hasta sexto grado). La ley 988 regía exclusivamente para las escuelas dependientes del gobierno provincial, que si bien eran mayoritarias (82\%), coexistían, a partir de la Ley Lainez ${ }^{11}$, con escuelas nacionales radicadas en la provincia (8\%), además de los colegios privados (10\%). Estos porcentajes tomados para 1946, guardan correlación con la dependencia institucional de la matrícula, los docentes y con la tendencia de los años previos (MEMORIA DEL CONSEJO NACIONAL DE EDUCACIÓN, 1946).

En 1905 la Ley de Educación Común fue reformada, reduciéndose a cuatro años la edad de escolaridad obligatoria con el argumento del bajo rendimiento del sistema educativo, causado por la deserción y el analfabetismo. La exigencia quedaba restringida a las escuelas elementales, incrementándose de esta forma, los contrastes entre las áreas urbanas y rurales, ya que hasta 1913 las escuelas complementarias pertenecieron exclusivamente a las primeras. Esta diferenciación, encontraba antecedentes en las condiciones y el funcionamiento de las escuelas, así como en el discurso de la Dirección General de Escuelas, en el cual se argumentaba que los establecimientos rurales debían tener una reglamentación específica que redujera los contenidos, los horarios de

\footnotetext{
${ }^{10}$ Véase ley 988 [en línea] http://www.gob.gba.gov.ar/dijl/DIJL/buscaid.php?var=3973.

${ }^{11}$ Según la Ley Láinez el Consejo Nacional de Educación establecería en las provincias que lo solicitaran escuelas elementales, infantiles, mixtas y rurales teniendo en cuenta el porcentaje de analfabetos. La ley surgió con el objeto de complementar el sistema educativo provincial y suplir las carencias existentes.
} 
la jornada, la edad escolar y que dejara de lado las pretensiones pedagógicas desmedidas (PINKASZ, 1993).

Entre 1921 y 1946, las escuelas dependientes del gobierno provincial pasaron de 1.695 a 2.460, dándose este crecimiento principalmente en la década del '20. Sin embargo, el ritmo en que fueron creados los establecimientos educativos, no alcanzó a igualar el incremento de la matrícula (Cuadro $\mathrm{n}^{\circ}$ ), por lo cual durante todo este período la proporción de alumnos por escuela se acrecentó con el transcurrir de los años, en un porcentaje similar al de todos los establecimientos de la nación. ${ }^{12}$ Como todos los promedios, estos datos no alcanzan a dar cuenta de la complejidad en la que convivían escuelas con matrícula muy diversa ${ }^{13}$, sin embargo nos permiten afirmar que el crecimiento de la población escolar era más veloz que el de las construcciones.

Cuadro 1 - Escuelas primarias y alumnos dependientes de la Dirección General de Escuelas de la provincia de Buenos Aires (1921-1946)

\begin{tabular}{|c|c|c|c|}
\hline Años & Escuelas & $\begin{array}{c}\text { Crecimiento } \\
\text { anual }\end{array}$ & $\begin{array}{c}\text { Alumnos por } \\
\text { escuela }\end{array}$ \\
\hline 1921 & 1.695 & - & 129 \\
\hline 1926 & 1.942 & 2.9 & 131 \\
\hline 1931 & 2.167 & 2.3 & 139 \\
\hline 1936 & 2.166 & 0.1 & 144 \\
\hline 1941 & 2.290 & 1.1 & 147 \\
\hline 1946 & 2.460 & 1.5 & 152 \\
\hline
\end{tabular}

Fuente: República Argentina (1946).

Las escuelas que estaban bajo la administración del gobierno de la provincia de Buenos Aires, presentaban una gran variedad tanto en el tipo de propiedad como en su estado de conservación. En 1946, el primer estado argentino contaba bajo su jurisdicción con un total de 2.460 escuelas, de las cuales sólo 833 eran fiscales, es decir un 34\% (DIRECCIÓN GENERAL DE ESCUELAS. Memoria, 1946). Estas fueron construidas a partir de planes de edificación realizados especialmente durante los gobiernos de Marcelino Ugarte (1902-1906), Valentín Vergara (19261930) y Manuel Fresco (1936-1940). Por lo tanto algunos edificios, tenían entre 20 y 40 años, cuando la matrícula provincial era mucho mas reducida. A esto se sumaba la falta de conservación de los mismos y la precariedad de los materiales de construcción. De las escuelas que no funcionaban en locales de propiedad fiscal, un $14 \%$ consistían en edificios cedidos

\footnotetext{
${ }^{12} \mathrm{El}$ crecimiento anual durante este período de los establecimientos escolares del país fue de un 1.8 y el de la matrícula de un 2.7 .

${ }^{13}$ Por ejemplo, en Bahía Blanca, en 1946, la escuela urbana número 2 contaba con más de 740 alumnos, mientras que a la escuela rural número 28 asistían solo cuarenta. (GOBIERNO DE LA PROVINCIA DE BUENOS AIRES, 1946).
} 
ubicados generalmente en sociedades de fomento y el $52 \%$ restante pagaba un alquiler que representaba una erogación anual de 1.270 .000 pesos. Mas allá del gasto, cabe suponer la inestabilidad que significaba que la mayor parte de las escuelas estuviera en manos privadas, tanto por la dificultad para realizar ampliaciones como por la posibilidad de que el dueño vendiera el local.

Durante el gobierno de Mercante, en el año 1948, se aprobó el Plan Integral de Edificación Escolar, por el que se invertían 600 millones de pesos para construir 1.500 escuelas. ${ }^{14}$ Si bien la gestión peronista no fue objeto de este trabajo, podemos plantear que el Plan de Edificación dio resultado (LONGONI, 2006). La Memoria del Ministerio de Educación de 1958 indica que para ese año las escuelas dependientes de la provincia eran 3.432, lo que implica un crecimiento de cerca de mil establecimientos desde 1946. Por otra parte, mientras que en vísperas del gobierno de Mercante, sólo un 34\% eran propiedad del estado, en 1958 esa cantidad había ascendido a más de un $70 \%$, los cedidos se mantuvieron en un $15 \%$, y los edificios alquilados se redujeron a un $12 \%$. Estas cifras parecen demostrar que durante estos años, más allá del incremento del número de establecimientos, fue de central importancia que gran parte de ellos pasaran a ser propiedad del Estado.

\section{El analfabetismo en la provincia de Buenos Aires: una mirada desde los discursos.}

Los resultados del Tercer Censo Nacional de Educación realizado en 1931, dieron lugar a una lectura pesimista respecto a los índices de analfabetismo, enmarcada en el contexto de la crisis del consenso liberal. ${ }^{15}$ Desde la publicación de sus resultados se elaboran una serie de diagnósticos y proyectos sobre el sistema educativo, tanto a nivel nacional como provincial. En 1933 el Consejo Nacional de Educación creó el Cuerpo de Maestros Inspectores de Obligación Escolar, cuyo objetivo era controlar el fiel cumplimiento de la asistencia, y al año siguiente se realizó la Primera Conferencia Nacional de Analfabetismo donde se establecieron consideraciones acerca de las trabas para su derrota. Si bien se tuvieron en cuenta factores vinculados al sistema educativo, se dio importancia principalmente a los condicionantes socioeconómicos tales como el trabajo infantil, la insatisfacción de las necesidades básicas o la inexistencia de caminos.

\footnotetext{
${ }^{14}$ Véase ley 5283 [en línea] http://www.gob.gba.gov.ar/dijl/DIJL/buscador.php?tipo=01.

${ }^{15}$ La visión critica respecto a la educación primaria cristalizada a partir de los resultados del Censo de 1931, no se redujo a las orientaciones de la matrícula sino también, a aspectos de tipo pedagógicos que cuestionaban el positivismo. (CARLI, 2002; PUIGGRÓS, 1992).
} 
En concordancia con la política nacional, el objetivo de desterrar el analfabetismo fue una de las prioridades del gobierno bonaerense a cargo de Manuel Fresco. En la apertura de sesiones legislativas del año 1936, planteaba como su principal preocupación en materia educativa combatir el analfabetismo y la deserción escolar. (Diario de Sesiones de la Cámara de Diputados, 1936, p. 20). Sin embargo, las propuestas, parecían buscar la solución presionando a la población escolar para que concurriera a los establecimientos educativos. Si bien complementariamente se realizaron obras de infraestructura y reformas institucionales, el acento fue colocado en aquellas medidas que a través de la coacción se proponían alfabetizar la población. Durante su segundo año de gobierno, Manuel Fresco sancionó la "Ley de Represión del Analfabetismo y Deserción Escolar", según la cual todo padre o encargado de menores estaba obligado a declarar la existencia de sus hijos o pupilos dentro de los seis meses cumplidos los cinco años de edad y en caso de no hacerlo debían pagar una multa. En su artículo 17 expresaba: "El alumno que no se presentase en el siguiente curso escolar o no pidiese pase a otro distrito, como también el que abandonase durante el período de enseñanza será considerado desertor escolar."16 Paralelamente se desarrolló una campaña provincial contra el analfabetismo que instituyó la "Semana del Analfabeto" (FRESCO, 1940, p. 146). Respecto a la población analfabeta adulta, el Inspector General de Escuelas del gobierno de Fresco, David Kraiselburd, propició una serie de medidas, como no licenciar a los conscriptos del Servicio Militar, no otorgar libertad condicional a los reclusos y prohibir el acceso al país, a quienes no supieran leer y escribir (PINEAU, 2002).

Diez años después de que Manuel Fresco presentara su proyecto educativo ante la Legislatura, el analfabetismo y el semianalfabetismo provocado por la deserción escolar continuaban siendo la principal preocupación de los gobiernos en materia educativa. La persistencia de la problemática en los discursos durante la década del `40 señala que las soluciones intentadas no dieron los resultados esperados, lo cual podemos suponer, guarda relación con dos rasgos de la política educativa hasta el momento. Por un lado, medidas que acentuaban las diferencias entre las áreas urbanas y rurales y por otra parte, la falta de concordancia entre los diagnósticos realizados y los proyectos, que pretendían dar solución al problema del analfabetismo ejerciendo presión sobre la población en edad escolar y el adulto analfabeto.

Las elecciones de febrero de 1946 que llevaron a la presidencia a Juan Domingo Perón, condujeron a Domingo Mercante como titular del Poder Ejecutivo bonaerense con el título de gobernador y dieron forma a la composición de la Legislatura provincial, integrada por una Cámara de Diputados, donde el oficialismo era mayoritario, y una Cámara de Senadores compuesta por el mismo número de oficialistas que de opositores. ${ }^{17}$ Una vez asumida la nueva

\footnotetext{
${ }^{16}$ Ley número 4546 [en línea] http://www.gob.gba.gov.ar/dijl/DIJL buscaid.php?var=6520. Por otra parte creaba los cargos de agentes escolares y la Oficina de Obligación Escolar que dependiendo de la Dirección General de Escuelas, tendría a su cargo el padrón escolar de cada distrito (Diario de Sesiones de la Cámara de Diputados, 1937, p. 2852-2853).

${ }^{17}$ Del total de diputados, 49, habían apoyado la candidatura de Mercante y 35 pertenecían al partido opositor, denominado Unión Cívica Radical, al cual comúnmente se refiere como radicalismo. La Cámara de Senadores, estaba compuesta por 21 miembros oficialistas y 21 opositores. Cabe aclarar que Mercante fue apoyado por diferentes partidos que presentaron
} 
gestión, Alejandro Greca, un abogado santafecino de orientación forjista fue designado en el cargo de Director General de Escuelas. ${ }^{18}$

En la apertura de sesiones legislativas de 1946 el gobernador Domingo Mercante expresaba que "Los problemas de la educación serán objeto de las mas cuidadosa preocupación de mi gobierno. No podía ser de otra manera en un Estado donde aun debe ser desarraigado el analfabetismo." (Diario de Sesiones de la Cámara de Diputados, 1946, p. 50). Rápidamente, tanto por parte del oficialismo como de la oposición, se presentaron numerosos proyectos que constituyeron una serie de fórmulas, que a partir de promover los medios adecuados, pretendieron contribuir a superar las trabas que impedían extender los índices de alfabetización. Si tomamos la lista de asuntos entrados en la Cámara de Diputados, encontramos que solo en 1946 fueron presentados más de cien proyectos en materia educativa, la mayor parte de los cuales consistían en pedidos particulares para ampliar, refaccionar o construir escuelas de un determinado distrito. Asimismo, se enviaron proyectos integrales entre los cuales cabe destacar, planes de edificación escolar, la creación de puestos de maestros, de escuelas volantes rurales, de comedores escolares, bibliotecas, unidades sanitarias, así como de facilidades para el transporte, ${ }^{19}$ algunos de los cuales fueron retomados de los gobiernos anteriores. ${ }^{20}$

Aunque no todos los proyectos se llevaron a cabo, la fundamentación de los mismos arroja luz acerca de las representaciones que tenían sus autores. Si bien se hizo referencia a la necesidad de reformar la Ley de Educación y los contendidos escolares ${ }^{21}$, en un primer momento las trabas que se plantearon para superar el problema del analfabetismo fueron la falta y mala distribución tanto de maestros ${ }^{22}$ como de edificios escolares, especialmente en los distritos rurales.

candidatos propios para el poder ejecutivo, por lo tanto las bancas oficialistas, estuvieron conformados hasta 1948, por miembros del Partido Laborista y de la Unión Cívica Radical Junta renovadora. Para más información sobre esta cuestión ver AELO (2004).

${ }^{18}$ Alejandro Greca, fue miembro del Instituto de Derecho Civil de la Facultad del Litoral y del Consejo Directivo de dicha facultad en diversos períodos, fue vicedecano en el año 1945 y miembro de la Convención Constituyente de la provincia de Santa Fe (DIRECCIÓN GENERAL DE ESCUELAS - Memoria, 1946).

${ }^{19}$ Entre mediados de 1946 y principios de 1947 se presentaron tres proyectos de carácter general con el objeto de construir y refaccionar escuelas en la provincia de Buenos Aires. Uno del diputado radical Juan Naim, otro del diputado laborista Francisco Bertrán y finalmente, un proyecto del Poder Ejecutivo elaborado por el director general de escuelas Alejandro Greca. Para tratar esta cuestión, en octubre de 1946 se constituyó una Comisión Especial de Construcciones Escolares. Otro ejemplo que podemos mencionar es el proyecto presentado por el diputado radical Vicente Musacchio para crear un centro de asistencia médica y social en cada distrito donde funcionase una escuela. (Diario de Sesiones de la Cámara de Diputados, 1946, p. 831).

20 Por ejemplo, el diputado laborista Yezid Yanzón, reprodujo dos proyectos anteriores. Uno presentado por el diputado conservador Mario Uzal, en julio de 1942 para crear becas para los alumnos que tuvieran las mejores calificaciones. El segundo del Poder Ejecutivo, presentado en mayo de 1943, para crear comedores escolares, (Diario de Sesiones de la Cámara de Diputados, 1946, p. 111-112).

${ }^{21}$ Una vez reformada la Constitución de la provincia de Buenos Aires, se realizaron varias propuestas para modificar la Ley de Educación Común. En julio de 1946, el diputado oficialista Adolfo Silvestre presentó un proyecto de "nueva estructuración y programas de enseñanza para los establecimientos dependientes de la Dirección General de Escuelas (Diario de Sesiones de la Cámara de Diputados, 1946, p. 723).

${ }^{22}$ Según los testimonios, la escasez de docentes era uno de los principales factores causantes de que se cerraran grados e incluso escuelas. Sin embargo, también fue señalada la existencia escuelas con una enorme cantidad de docentes y de maestras sin grado, aglutinadas en establecimientos donde desempeñaban funciones en otros cargos, especialmente 
En este sentido, el Director General de Escuelas del gobierno de Mercante, Alejandro Greca señalaba: “...existían locales que en su época fueron considerados como monumentales y hoy están totalmente destruidos, al extremo de que la refacción costaría mas que la construcción del nuevo edificio" (Revista de Educación, 1946, p. 89-90). Coincidiendo con esta apreciación, un diputado perteneciente a la Unión Cívica Radical (UCR) Juan Naim, señalaba que a pesar de la riqueza económica de la provincia el $\mathbf{8 0} \%$ de las escuelas se encontraban en mal estado. Según esta repartición la casi totalidad de los edificios alquilados, se encontraba "en un estado ruinoso e inhabitable que no responde a las exigencias y necesidades mas elementales de la instrucción primaria" (Dirección General De Escuelas. Memoria, 1946).

Pocos días después de asumida la nueva gestión, tres senadores pertenecientes a la $\mathrm{UCR}^{23}$ presentaron un proyecto de minuta de comunicación sobre datos referentes a la Dirección General de Escuelas. En esa oportunidad tanto los miembros de su partido como los del oficialismo manifestaron varias declaraciones respecto al estado en que se encontraba la educación en ese entonces, y describieron el escenario de los colegios de sus distritos. El senador oficialista Luis Passerini señalaba: "Y las escuelas rurales casi ninguna tiene edificio, algunas funcionan en ranchos y se da el caso de una en la Colonia de Bellocq, que no tiene edificio, pues se da clase al aire libre" (Diario de Sesiones de la Cámara de Senadores, 1946, p. 264). En la misma sesión, el senador de la oposición Alfredo Laffaye expresaba: "En Veinticinco de Mayo, la escuela número 9, sobre una inscripción de cincuenta alumnos, tenía una sola maestra para los siete años teóricos, porque en realidad solo pueden concurrir los niños hasta tercer grado" (Diario de Sesiones de la Cámara de Senadores, 1946, p. 257). La lectura de los informes de los inspectores para las escuelas de la provincia de Buenos Aires durante 1946, así como los datos acerca de la matrícula y el número de docentes confirman estas afirmaciones (Estadística Mensual de las Escuelas Públicas de la Provincia de Buenos Aires, 1946).

Si bien la evaluación de la situación escolar elaborada apenas asumida la gestión peronista, no discrepaba de aquella expresada durante la década del '30, la diferencia se encuentra en la búsqueda de las responsabilidades. Mientras que los diagnósticos realizados en los años '30 consideraban que la problemática del analfabetismo residía en factores externos al sistema educativo y que la solución consistía en ejercer mayor coerción sobre la población escolar, la reciente gestión buscó la responsabilidad en el deficiente funcionamiento de la Dirección General de Escuelas. Por ejemplo, la ley de Registro de Edad Escolar ${ }^{24}$ sancionada en 1947, cuyo objetivo era saber con exactitud la verdadera concurrencia escolar así como los lugares en los que abundaba la deserción y sus causas, para combatir en forma enérgica el analfabetismo (Diario de Sesiones de la Cámara de Senadores, 1946, p. 60), contrastaba con la ley de Represión del Analfabetismo del gobierno de Fresco. En este sentido nos parece interesante destacar el

${ }^{23}$ Se trataba de Alfredo Laffaye, Vicente Biscayart y Federico Cané.

${ }^{24}$ Véase ley 5181 [en línea] http://www.gob.gba.gov.ar/dijl/DIJL_buscaid.php?var=6952. 
planteo de Sandra Carli, respecto a los cambios sobre la concepción de la niñez durante el peronismo. Según la autora, "la infancia comenzaba a ligarse con una exigencia de inscripción de la niñez en una sociedad de masas, y en ese sentido, con un reclamo de participación ciudadana y con una experiencia de mayor autonomía" (CARLI, 2002).

Tanto el peronismo como la oposición centraron su crítica en las gestiones anteriores. En el primer caso, es cierto que podría verse como parte de la ofensiva al pasado que el peronismo era llamado a modificar. En este sentido, cabe señalar el discurso del senador laborista Benito Ferro: “Todos los gobiernos hicieron su campaña política a costa de la enseñanza escolar... Hoy creemos sinceramente en este gobierno. Y creemos en el nuevo director de Escuelas que subsanará todos los errores..." (Diario de Sesiones de la Cámara de Senadores, 1946, p. 261). La oposición sin más posibilidad ante la novedad del gobierno peronista, no perdió oportunidad de referirse a la gestión de la Revolución de Junio. En este sentido el diputado Romariz Elizalde expresaba: "El gobierno legal al hacerse cargo de la cosa pública en la provincia, se ha encontrado con la Dirección General de Escuelas en un estado de descomposición que ha impresionado a la opinión pública y que es consecuencia de gobiernos anteriores incluso desde luego, de los gobernantes del gobierno de facto..." (Diario de Sesiones de la Cámara de Diputados, 1946, p. 11081110). Sea como fuere, existió un amplio consenso en que la situación de anarquía y desgobierno existente en la Dirección General de Escuelas, resultaba inadecuada para abordar los factores que incidían en el analfabetismo.

Ahora bien, ¿a qué se referían los dirigentes políticos al expresar que el caos y la anarquía de la Dirección General de Escuelas dificultaban la creación de establecimientos escolares y puestos de maestros? En primer lugar, se planteó la variabilidad del personal, sumada a los puestos vacantes. Entre 1940 y 1946, en la Dirección General de Escuelas, se sucedieron doce directores, dieciséis secretarios, seis oficiales mayores, cuatro inspectores y cinco subinspectores. En lo que respecta al Consejo General de Educación, durante ese período raras veces el número de consejeros escolares llegó a ocho, incluso de agosto a octubre de 1946 los cargos estuvieron desiertos esperando designación. Por otra parte, los integrantes de la mesa directiva (vicepresidente primero y segundo) variaron diez veces su composición. Si bien, la oposición, acusaba de esta situación, al continuo cambio de los directores generales (Diario de Sesiones de la Cámara de Senadores, 1946, p. 257), la coalición peronista, sin embargo, no fue homogénea respecto a esta cuestión. Mientras que el sector perteneciente a la UCR Junta Renovadora (Diario de Sesiones de la Cámara de Senadores, 1946, p. 259) coincidía con este planteo, el laborismo hacía referencia a la responsabilidad de los cuadros técnicos (Diario de Sesiones de la Cámara de Senadores, 1946, p. 265).

En segundo lugar fue señalado el desconocimiento del estado general de la educación para poder construir escuelas y designar maestros en los distritos donde realmente hiciera falta. A menos hasta la publicación del Censo General de 1947, contaban con los datos del Cuarto Censo 
Nacional de Educación de 1943, recién publicado en 1948, y expresaban por ello un malestar. A su vez, la escasez de información señalada, era un argumento para justificar la afluencia de pedidos en particular para los distritos de origen de los legisladores, que se dio durante los primeros meses de la gestión legislativa, cuya información afirmaban diputados y senadores, conseguían por cuenta propia. En este sentido, y en el marco de la discusión del proyecto de ley sobre la creación de un registro de escolar, el senador laborista Edmundo Vampa, planteaba que "Los censos que a tal efecto se han realizado, no han rendido resultados prácticos, desconociéndose las verdaderas necesidades escolares de la provincia." (Diario de Sesiones de la Cámara de Senadores, 1946, p. 1776).

En tercer lugar, otros testimonios que daban cuenta del funcionamiento de la Dirección General de Escuelas, se referían a la falta de administración de los recursos. Por ejemplo, en un comunicado emitido por la nueva gestión educativa, sobre la recuperación de material escolar deteriorado que fuera adquirido en 1937 con destino "a una proyectada reforma educativa. Fracasada esta supuesta reforma", señalaba que "buena parte de él ha sido distraído de la aparente finalidad educativa que se invocó al distribuirlo y aplicado a distintos usos" (Boletín Oficial de Educación, 1946). En este sentido, el Director General de Escuelas expresaba que contaba con un millar de terrenos para construir escuelas donados en distintas épocas, que habían sido "abandonados o desconocidos la mayoría hasta la fecha, por la falta de interés de anteriores autoridades”. (DIRECCION GENERAL DE ESCUELAS. Memoria, 1946).

Finalmente, los legisladores resaltaban la existencia de cierta superposición de potestades entre la autárquica Dirección General de Escuelas, y por un lado el Ministerio de Gobierno del que formaba parte, y por otro lado, la legislatura. El senador opositor Alfredo Laffaye parecía indicarlo en los siguientes términos "lo que yo quiero pedir con esta minuta... es que venga el señor Ministro, que venga el director de escuelas o quien corresponda y nos explique la razón de estas fallas...." (Diario de Sesiones de la Cámara de Senadores, 1946, p. 258). Las cuestiones que dieron mayor espacio a estas superposiciones fueron aquellas referidas a la elección sobre las localidades donde construir escuelas y la designación de los puestos de maestros creados. ${ }^{25}$

Si bien existió consenso en la evaluación crítica del gobierno educativo heredado, no hubo acuerdo en como solucionar su situación. Los legisladores de la oposición pretendían descentralizar el gobierno educativo, dándoles a los Consejos Escolares la autoridad que habían perdido en 1905. En efecto, expresaron que los gobiernos locales eran los verdaderos representantes populares ante la falta de comprensión por parte de la dirección central de los

\footnotetext{
${ }^{25}$ La creación de nuevos puestos de maestros por parte de la legislatura, deja entrever el debate sobre si correspondía a la Dirección General de Escuelas o si era atribución de legisladores establecer que los docentes seleccionados fueran domiciliados en el lugar donde debían llenarse las vacantes, y solo en el caso de que no los hubiera con los de otras localidades. También al tratarse la distribución de diez millones de pesos para la adquisición y/o expropiación de terrenos para edificaciones escolares en la zona del Gran Buenos Aires, se debatió si correspondía al Poder Ejecutivo o a la Dirección General de Escuelas realizar las distribuciones de dinero según las localidades. Véase Diario de Sesiones de la Cámara de Senadores, (1947, p. 2710-2713 y 1948, p. 335-356).
} 
problemas del sistema educativo (Diario de Sesiones de la Cámara de Senadores, 1946, p. 263). El oficialismo, por el contrario, en un contexto en que el Estado estaba aumentando su ingerencia, consideraba la autarquía de la Dirección General de Escuelas y el gobierno local de los Consejos Escolares como un impedimento. Sin embargo debieron esperar a la Reforma Constitucional realizada en 1949 para reemplazar la Dirección General de Escuelas, el Consejo General y los Consejos Escolares de distrito por un departamento de Estado dependiente directamente del Poder Ejecutivo.

\section{Un escenario heterogéneo: los índices de analfabetismo en la provincia de Buenos Aires.}

El sistema educativo argentino desde el punto de vista cuantitativo ha sido estudiado por varios investigadores. Entre ellos Juan Carlos Tedesco (1986) da cuenta de las dificultades para precisar la relación entre matrícula, población escolar y analfabetismo a lo largo el tiempo, debido a la carencia de datos, unida a la dudosa confiabilidad de los existentes. Esta situación es compartida para el estudio de la provincia de Buenos Aires, lo cual no imposibilita tomar las cifras como indicativas de tendencias, sin perder de vista los matices entre las diferentes fuentes.

Cuadro 2 - Matrícula de escuela primaria en el territorio de la provincia de Buenos Aires (19211946)

\begin{tabular}{|c|c|c|}
\hline Año & Matrícula & $\begin{array}{c}\text { Crecimiento } \\
\text { anual }\end{array}$ \\
\hline 1921 & 284.449 & - \\
\hline 1926 & 322.837 & 2.7 \\
\hline 1931 & 372.714 & 3.1 \\
\hline 1936 & 389.312 & 0.9 \\
\hline 1941 & 421.019 & 1.6 \\
\hline 1946 & 464.003 & 2.4 \\
\hline
\end{tabular}

Fuente: República Argentina (1946).

Entre 1921 y 1946 la matrícula escolar de la provincia de Buenos Aires se incrementó en 179.554 alumnos. Este crecimiento se dio principalmente en la década del '20, produciéndose una ralentización a partir de 1930, que en el quinquenio siguiente comenzó a recuperarse. Es difícil comparar con datos similares para la población en edad escolar, ya que los Censos de 1943 y 1947 presentan cierta discrepancia en su información. De todas formas, tomando datos generales de población podemos afirmar que si bien durante este período el crecimiento poblacional fue 
superior al incremento de la matrícula escolar, ${ }^{26}$ en vísperas del peronismo la matrícula de enseñanza primaria de los niños entre 6 y 13 años superaba el $70 \%{ }^{27}$

Cuadro 3 - Porcentaje de analfabetismo en la población de 14 y más años, clasificada por grupos de edad en la provincia de Buenos Aires (1947).

\begin{tabular}{|c|c|}
\hline Grupo de edades & Buenos Aires \\
\hline Mayor de 14 años. & $9.8 \%$ \\
\hline De 14 a 29 años. & $3.9 \%$ \\
\hline De 30 a 49 años. & $8.3 \%$ \\
\hline De 50 y más años. & $22.0 \%$ \\
\hline
\end{tabular}

Fuente: Presidencia de la Nación (1947, t. 1, v. 1).

Teniendo en cuenta la dificultad de establecer una causalidad lineal entre aumento de inscripción y alfabetización, analizar los datos acerca del analfabetismo a través del tiempo, nos permite en cierto modo realizar una aproximación a los resultados del sistema educativo primario en los años previos al gobierno peronista. Según vemos en el cuadro $\mathbf{n}^{\circ} 3$, el índice de analfabetismo en las personas mayores de 14 años se incrementa con la edad. ${ }^{28} \mathrm{Si}$ consideramos que la instrucción primaria en la provincia de Buenos Aires comenzaba a partir de los ocho años, podemos suponer que el mayor descenso del analfabetismo se habría dado entre quienes asistieron a la escuela durante el período 1906 - 1925 (edades de 30 a 49 años), coincidiendo con la incorporación de los hijos de inmigrantes al sistema educativo. En el período siguiente la acentuación de las inmigraciones internas, así como los cambios sociales relacionados con la política y la economía a partir de 1930 habrían ralentizado ese ritmo.

La heterogeneidad de la distribución poblacional de la provincia se trasladaba al mapa escolar y así por ejemplo, en 1947, mientras que en el distrito urbano de Vicente López, cinco de cada cien niños no sabían leer ni escribir, lo cual se asemejaba al escenario que en ese entonces presentaba la Capital Federal, en el distrito rural de Tordillo, ese número se ampliaba considerablemente elevándose a veintiún niños, recordando en este caso la situación de la provincia de Tucumán. Si reparamos en el grado de analfabetismo de cada distrito, la situación también resulta dispar. Mientras que solo en catorce, el índice se encontraba por debajo de la media urbana $(8.1 \%)^{29}$, en otros veintisiete, el porcentaje superaba el promedio rural provincial

\footnotetext{
${ }^{26}$ Entre 1914 y 1947 la población de la provincia de Buenos Aires se duplicó, pasando de 2.066.165 a 4.272.337 habitantes.

${ }^{27}$ El Censo Escolar de 1943 señala para el nivel primario de todo el país una tasa de escolarización del 75.3\% y para la provincia de Buenos Aires de un 71.3\%. En cambio, los datos aportados por el Censo Nacional de Población, para 1947 arrojan una tasa menor para el país de un $73.5 \%$ y para la provincia de Buenos Aires de un 77.7\%, mientras que en 1914 era de un $48.8 \%$ y $42.9 \%$ respectivamente. (IV Censo General de la Nación, 1947; IV Censo Escolar de la Nación, 1948).

${ }^{28}$ Según señala el Censo de 1947, "no se puede considerar a nadie como analfabeto mientras no haya sobrepasado la edad de la instrucción primaria obligatoria _ que va de los seis a los trece años de edad". Cabe aclarar que en la provincia de Buenos Aires, la obligatoriedad escolar se había reducido de los ocho a los doce años.

${ }^{29}$ Se trataba de los distritos de Vicente López (5.2\%), General Pueyrredón (6.3\%), General Sarmiento (6.4\%), La Plata (6.4\%), San Isidro (6.6\%), Coronel Rosales (6.6\%), Bahía Blanca (6.9\%), Morón (6.9\%), Quilmes (6.9\%), San Fernando (6.9\%), Almirante Brown (7.1\%), General San Martín (7.4\%) Cuatro de Junio (8.0\%), Marcos Paz (8.1\%).
} 
$(14.2 \%)^{30}$

Los contrastes se presentaban incluso dentro de los mismos distritos. Si bien en la provincia de Buenos Aires, no era tan acentuado como en el país (Cuadro $n^{\circ} 4$ ), tomando los datos desagregados de cada uno de los ciento doce distritos podemos dar cuenta de las diferencias internas. Así por ejemplo, si en la media provincial la diferencia entre la zona urbana y rural era del 6.1\%, en diecinueve distritos esta distancia era mayor. (Cuadro $n^{\circ} 4$ ). Esta situación se daba tanto en zonas rurales con un alto índice de analfabetismo (por ejemplo, Rojas y Salto), como aquellas cercanas a la Capital Federal, (por ejemplo, Morón, Florencio Varela, Lomas de Zamora) y hasta incluso en la Capital de la Provincia.

Cuadro 4 - Porcentaje de analfabetismo en la población de 14 y más años de edad, clasificada por jurisdicción y zona en que vive en la provincia de Buenos Aires (1947).

\begin{tabular}{|c|c|c|c|c|c|}
\hline \multirow{2}{*}{ Jurisdicción } & \multicolumn{2}{|c|}{ Zona Urbana } & \multicolumn{2}{c}{ Zona Rural } & \multirow{2}{*}{ Diferencia } \\
\cline { 2 - 5 } & $\begin{array}{l}\text { Total de } \\
\text { población }\end{array}$ & $\begin{array}{l}\text { Analfabetismo } \\
\text { (en \%) }\end{array}$ & $\begin{array}{l}\text { Total de } \\
\text { población }\end{array}$ & $\begin{array}{l}\text { Analfabetismo } \\
\text { (en \%) }\end{array}$ & \\
\hline Argentina & 7.504 .552 & $8.8 \%$ & 3.817 .344 & $23.2 \%$ & $14.4 \%$ \\
\hline Buenos Aires & 2.323 .359 & $8.1 \%$ & 893.008 & $14.2 \%$ & $6.1 \%$ \\
\hline L. de Zamora & 99.237 & $8.4 \%$ & 1.423 & $22.4 \%$ & $14.0 \%$ \\
\hline Zarate & 26.406 & $11.0 \%$ & 3.801 & $23.8 \%$ & $12.8 \%$ \\
\hline Lujan & 14.457 & $7.6 \%$ & 15.327 & $17.0 \%$ & $10.6 \%$ \\
\hline Pergamino & 24.798 & $9.2 \%$ & 29.089 & $19.1 \%$ & $9.9 \%$ \\
\hline Salto & 5.940 & $11.2 \%$ & 14.391 & $20.4 \%$ & $9.2 \%$ \\
\hline Gral. Alvear & 1.755 & $12.9 \%$ & 4.126 & $21.5 \%$ & $8.6 \%$ \\
\hline Rojas & 5.052 & $11.4 \%$ & 16.966 & $19.9 \%$ & $8.5 \%$ \\
\hline Merlo & 10.694 & $6.5 \%$ & 4.526 & $14.8 \%$ & $8.3 \%$ \\
\hline Gral. Alvarado & 2.654 & $6.3 \%$ & 6.575 & $14.5 \%$ & $8.2 \%$ \\
\hline 25 de Mayo & 8.767 & $11.7 \%$ & 19.582 & $19.4 \%$ & $7.7 \%$ \\
\hline B. Mitre & 8.679 & $10.7 \%$ & 15.076 & $18.2 \%$ & $7.5 \%$ \\
\hline La Plata & 214.070 & $5.7 \%$ & 22.960 & $13.1 \%$ & $7.4 \%$ \\
\hline Patagones & 4.084 & $7.9 \%$ & 6.814 & $15.2 \%$ & $7.3 \%$ \\
\hline Campana & 11.416 & $8.1 \%$ & 2.187 & $15.3 \%$ & $7.2 \%$ \\
\hline Dolores & 10.295 & $9.0 \%$ & 3.698 & $15.9 \%$ & $6.9 \%$ \\
\hline Gral. Villegas & 3.496 & $7.5 \%$ & 14.944 & $14.4 \%$ & $6.9 \%$ \\
\hline F. Varela & 4.329 & $6.2 \%$ & 3.599 & $12.9 \%$ & $6.7 \%$ \\
\hline Las Conchas & 19.016 & $8.3 \%$ & 24.615 & $14.5 \%$ & $6.2 \%$ \\
\hline Moreno & 5.680 & $5.7 \%$ & 5.306 & $11.8 \%$ & $6.1 \%$ \\
\hline & & & & & \\
\hline
\end{tabular}

Fuente: Presidencia de la Nación (1947, t. 1, v. 1).

\footnotetext{
${ }^{30}$ Baradero (21.3\%), Tordillo (21.1\%), General Alvear (18.9\%), General Viamonte (18.4\%), Rojas (17.9\%), Salto (17.7\%), General Arenales (17.4\%), General Madariaga (17.2\%), Veinticinco de Mayo (17.0\%), General Lavalle (16.9\%), Roque Pérez (16.3\%), Saladillo (16.3\%), Caseros (16.2\%), Ramallo (15.8\%), Nueve de Julio (15.6\%), Bartolomé Mitre (15.5\%), Chacabuco (15\%), Balcarce (14.9\%), Adolfo Alsina (14.7\%), Colón (14.7\%), Pergamino (14.6\%), San Antonio de Areco, (14.4\%), Leandro N. Alem (14.4\%), General Pinto (14.4\%), Peguajó (14.2\%), Carlos Casares (14.2\%), Bragado (14.2\%).
} 
Al igual que al referirnos a la población urbana y rural, el índice de analfabetismo desagregado por género muestra mayor contraste en el país que en la provincia de Buenos Aires (Cuadro $\mathrm{n}^{\circ}$ 5). La diferencia media entre varones y mujeres (1.1\%), se acentúa en la población urbana, en la cual el analfabetismo en las mujeres es de un $9.4 \%$ y en los hombres de un $6.7 \%$, y se revierte en la población rural, en la cual el analfabetismo en las mujeres es inferior (13.4\%) que en los varones (14.7\%), lo cual puede guardar relación con la temprana incorporación de los hombres a las actividades rurales. Cuando observamos esta variable desagregada por distritos, encontramos que la mayoría de los casos se encuentran dentro de la media.

Cuadro 5 - Porcentaje de analfabetismo en la población femenina y masculina de 14 y más años de edad en el país y Buenos Aires (1947).

\begin{tabular}{|c|c|c|c|}
\hline Jurisdicción & Total & Hombres & Mujeres \\
\hline Total del país & $13.6 \%$ & $12.1 \%$ & $15.2 \%$ \\
\hline Buenos Aires & $9.8 \%$ & $9.3 \%$ & $10.4 \%$ \\
\hline
\end{tabular}

Fuente: Presidencia de la Nación (1947. t. 1, v. 1).

La situación se modifica, cuando consideramos las diferencias de género en relación a las franjas etáreas. En el grupo de población de 14 a 29, el porcentaje de mujeres analfabetas es menor al de los hombres por un margen no muy amplio ( $3.2 \%$ y $4.6 \%$ respectivamente). En el grupo entre 30 y 49 años de edad, la diferencia se acota aún mas, pero la relación es inversa (8.6\% y $8 \%$ respectivamente). Sin embargo, en el caso de la franja etárea de 50 años en adelante, el porcentaje de mujeres analfabetas es mucho mayor al de los hombres (26\% y $18.7 \%$ respectivamente). Esto prueba la importancia del proceso de escolarización de las mujeres en el descenso del analfabetismo que se da en la población entre 30 y 49 años.

Por otra parte, mas allá de quienes eran considerados analfabetos, cabe mencionar la problemática del semianalfabetismo, es decir, aquellos sabiendo leer no escribían. Esta cuestión se encuentra relacionada con la deserción escolar (PUIGGRÓS, 1993; PINKASZ, 1997). En este sentido, tomamos los datos del Censo Educacional de 1943, a pesar de que su metodología permite presentar ciertas dudas sobre la exactitud de los datos, como un indicador de la situación. El mismo, señala que entre la población de 14 a 21 años que asistió a la escuela primaria, (438.010 alumnos), más del $60 \%$ interrumpieron el curso ${ }^{31}$. Este porcentaje, difiere en relación al último año aprobado, dándose el principal índice de deserción entre $3^{\circ}$ y $4^{\circ}$ grado, lo cual coincide con la reducción de la edad de obligatoriedad escolar a partir de $1905^{32}$.

\footnotetext{
${ }^{31}$ Al parecer, entre las razones de esta situación el Censo Escolar señala solo un factor relacionado con el sistema educativo: la falta de oferta escolar, el resto se trata de causas exógenas al mismo tales como trabajo, pobreza, enfermedad y distancia.

${ }_{32}^{2}$ La reforma de la Ley de Educación de 1905 reducía la obligatoriedad escolar a cuatro años (PINKASZ, 1993).
} 
Cuadro 6 - Población de 14 a 21 años que concurrió a la escuela primaria, clasificada según haya terminado o interrumpido el curso primario (1943).

\begin{tabular}{|l|l|l|l|}
\hline \multirow{2}{*}{ Jurisdicción } & Población & \multicolumn{2}{|l|}{ Interrumpieron el curso } \\
\cline { 3 - 4 } & de 14 a 21 años & Número & Porcentaje \\
\hline Provincia de Bs. As. & 438.010 & 263.498 & $60.2 \%$ \\
\hline Vicente López & 10.147 & 3.216 & $31.7 \%$ \\
\hline Lomas de Zamora & 12.908 & 4.589 & $35.5 \%$ \\
\hline General Conesa & 212 & 202 & $95.3 \%$ \\
\hline General Lavalle & 412 & 384 & $93.2 \%$ \\
\hline
\end{tabular}

Fuente: Presidencia de la Nación (1948, t. 1).

Si precisamos estos datos en los diferentes distritos de la provincia de Buenos Aires, surgen nuevamente importantes contrastes. Solo en 13 de ellos, más de la mitad de la población entre 14 y 21 años concluyó el curso primario, mientras que en veintitrés interrumpieron sus estudios más del $\mathbf{8 0} \%$. La diferencia entre zonas urbanas y rurales vuelve a influir en estos resultados. En el primer caso, se trata de distritos con una densidad de población, que en ese momento oscilaba entre los 50 y los 5.000 habitantes por kilómetro cuadrado, mientras que en el segundo caso, la fluctuación era entre 1 y 16 habitantes por kilómetro cuadrado. Como ejemplos extremos podemos mencionar por un lado los casos de Vicente López y Lomas de Zamora, y por otra parte los distritos de General Conesa y General Lavalle (Cuadro $\mathrm{n}^{\circ}{ }^{6}$ ).

En síntesis, podría decirse que si bien hacia 1947 el índice de analfabetismo para la provincia de Buenos Aires, había descendido de las dos cifras, se expresó de manera diversa en las distintas zonas de la provincia. Esto parece indicar entonces, que en el primer estado argentino convivían junto a distritos que se recordaban a la vecina Capital Federal, otros que se asemejaban a las provincias del interior de escasos recursos. Así lleva a comprender la preocupación que aún en la década del ' 40 permanecía vigente en los diferentes gobiernos de turno. Si bien la gestión peronista no fue objeto de este trabajo, podemos señalar para finalizar, que hacía 1960 el analfabetismo en la provincia de Buenos Aires se había reducido a un 5.6\% (V Censo General de la Nación, 1961) y que los dirigentes políticos en la década del '50 expresaban que había dejado de ser la preocupación primordial en materia educativa. Esto puede no ser sorprendente proviniendo de los mismos peronistas, sin embargo, el gobernador Oscar Alende al asumir su cargo, aunque queriéndole otorgar a su gobierno cierto carácter innovador, expresaba: "Superando su tradicional misión alfabetizadora, que será mantenida y ampliada, la escuela abarcará la educación sanitaria, física, moral, técnica y estética, de toda la comunidad que la circunda." (Diario de Sesiones de la Cámara de Diputados, 1958, p. 32). 


\section{Reflexiones finales}

Si tomamos los promedios estadísticos, pareciera que hacia 1947 el problema del analfabetismo formaba parte de una preocupación del pasado, sin embargo, constituía una constante en los discursos de los dirigentes políticos que se referían al sistema educativo. Al profundizar en las diferencias regionales, podemos señalar la disparidad existente entre los distritos con mayoría de población urbana y aquellos con baja densidad de población, pero también al interior de los mismos, constituyendo así un escenario heterogéneo similar al que podía encontrarse al interior del país. Por otra parte, si bien el índice de analfabetismo descendía junto a la edad de la población, la deserción que también presentaba amplias disparidades regionales, mantenía vigente la preocupación por aquella población que si bien estaba alfabetizada, no había completado su formación escolar.

Los contemporáneos hicieron de esta problemática el centro de sus discursos en materia educativa e incluso durante la década del '30 se realizó un Congreso de Analfabetismo. Tanto a nivel nacional como provincial los proyectos para modificar esta situación realizados en base a los diagnósticos elaborados, se centraron en una serie de medidas que a través de la coerción combinada con la asistencia social, pretendían disminuir el índice de analfabetismo. Sin embargo las acciones llevadas a cabo durante los años '30 en esta materia no tuvieron el impulso suficiente para mantener el ritmo de crecimiento de los años previos.

Al asumir la gestión peronista, el oficialismo y la oposición describieron un escenario educativo en el que tanto el mal estado como la necesidad de nuevas escuelas, así como la escasez e irregular distribución de docentes, hacían necesario una urgente reforma. En un primer momento la reforma educativa, mas que centrarse en cambios respecto al enfoque de la educación, se concentró en la necesidad de hacer mas eficiente el funcionamiento de la Dirección General de Escuelas, lo cual podemos suponer guarda relación con la pretensión por parte del gobierno peronista, tanto en el ámbito nacional como provincial, de reformar y hacer mas eficiente el estado.

En síntesis, el escenario educativo en vísperas del gobierno peronista de Domingo Mercante da cuenta de situaciones más complejas de lo que una primera visión pudiera apreciar. A mediados de la década del '40 el analfabetismo continuaba constituyendo una preocupación fundamental especialmente en las áreas rurales. A partir de las estadísticas posteriores, así como de la disminución de la centralidad que esta cuestión tuvo en los discursos de mediados de la década del '50, podemos sostener que el gobierno peronista de la provincia de Buenos Aires supuso un cambio significativo en la política educativa. 


\section{Documentos Oficiales}

GOBIERNO DE LA PROVINCIA DE BUENOS AIRES. Revista de Instrucción Primaria. 1941. _. DIRECCIÓN GENERAL DE ESCUELAS. Memoria. 1946. . DIRECCIÓN GENERAL DE ESCUELAS. Revista de Educación. La Plata, 1946. . BOLETÍN OFICIAL DE EDUCACIÓN. La Plata, Taller de Impresiones Oficiales 1940-1946. . ESTAdÍsTiCA MENSUAL DE LAS ESCUELAS PÚBLICAS DE LA PROVINCIA DE BUENOS AIRES. 1946.

. MINISTERIO DE EDUCACIÓN. Dirección de Personal y Estadística. Memoria año 1958.

_. _ . Diario de Sesiones de la Cámara de Diputados (DSCD). La Plata, Taller de Impresiones Oficiales $(1936,1937,1942,1946,1958)$.

. Diario de Sesiones de la Cámara de Senadores. (DSCS). La Plata, Taller de Impresiones Oficiales $(1946,1947,1948)$.

FRESCO, Manuel. La instrucción primaria en mi gobierno 1936-1940. Buenos Aires, Talleres Gráficos Daimano, 1940.

PRESIDENCIA DE LA NACIÓN. Secretaria Técnica. IV Censo Escolar de la Nación. Buenos Aires, 1948. t. 1.

_ _ _ _ _ . SECRETARIA TÉCNICA. IV Censo General de la Nación. Buenos Aires, 1947. t. 1, v. 1.

Buenos Aires, 1961.

DIRECCIÓN NACIONAL DE ESTADÍSTICAS Y CENSOS. $V$ Censo General de la Nación.

MINISTERIO DE EDUCACIÓN. DIRECCIÓN GENERAL DE ENSEÑANZA PRIMARIA. Educación Común en la capital, territorios y provincias. Talleres gráficos de Consejo Nacional de Educación, años 1940 y 1946.

\section{Referências}

ACHA, Omar; QUIROGA, Nicolás. Pliegues de la normalización de los estudios sobre el peronismo: complementos y aclaraciones. In: REIN, Raanan et al. Los estudios sobre el primer peronismo, aproximaciones desde el siglo XXI. La Plata: Instituto Cultural de la Provincia de Buenos Aires, 2009. p. 81-102.

AELO, Oscar. El peronismo en la legislatura de Buenos Aires: prácticas y concepciones políticas, 1946-1950. In: JORNADAS DE INVESTIGADORES DEL DEPARTAMENTO DE HISTORIA, 5., 2004, Mar del Plata. Anales... Mar del Plata: Universidad Nacional de Mar del Plata, septiembre de 2004. 
BARBA, Fernando; DEMARÍA, M. Elena. La provincia de Buenos Aires 1910-1987. La Plata: Archivo Histórico de la Provincia de Buenos Aires, 1997.

CARLI, Sandra. Niñez, pedagogía y política: transformaciones de los discursos acerca de la infancia en la historia de la educación argentina entre 1880 y 1955. Buenos Aires: Miño y Dávila, 2002.

CIAPPINA, Carlos. La gobernación de Domingo Mercante y las transformaciones estructurales del Estado bonaerense. In: PANELLA, Claudio (Comp.). El gobierno de Domingo A. Mercante en Buenos Aires (1946-1952): un caso de peronismo provincial. La Plata: Instituto de Cultura, Archivo Histórico de la Provincia, 2007. t. 3.

GERMANI, Gino. Estructura social de la Argentina. Buenos Aires: Solar, 1987.

HIRSCHEGGER, Ivana. La enseñanza primaria en el marco del Estado de Bienestar: los planes de obras y la expansión de los servicios educativos en dos municipios de Mendoza/Argentina (19461955). Revista Antíteses, Londrina, v. 3, n. 6, jul./dez. 2010. Disponible en: <http://www.uel.br/revistas/uel/index.php/antiteses>. Acceso en: 12 ago. 2011.

LONGONI, R. et al. Mercante y la infraestructura escolar: de la escuela palacio a la escuela comunidad. In: PANELLA, Claudio (Comp.). El gobierno de Domingo A. Mercante en Buenos Aires (1946-1952): un caso de peronismo provincial. La Plata: Instituto de Cultura, Archivo Histórico de la Provincia, 2006. t. 2.

MACOR, Darío; TCACH, César (Comp.). La invención del peronismo en el interior del país. Santa Fe: Universidad Nacional del Litoral, 2003.

MELÓN PIRRO, Julio; QUIROGA, Nicolás (Comp.). El peronismo Bonaerense. Mar del Plata: Suárez, 2006.

PANELLA, Claudio (Comp.). El gobierno de Domingo A. Mercante en Buenos Aires (1946-1952): un caso de peronismo provincial. La Plata: Instituto de Cultura, Archivo Histórico de la Provincia, 2005.

PINEAU, Pablo. Para qué enseñar a leer. In: CUCCUZZA, Héctor; PINEAU, Pablo. Para una historia de la enseñanza de la lectura y la escritura en Argentina: del catecismo colonial a La Razón de Mi Vida. Buenos Aires: Miño y Dávila, 2002.

PINKASZ, Daniel. Escuelas y desiertos: hacia una historia de la educación primaria de la provincia de Buenos Aires. In: PUIGGRÓS, Adriana; OSSANNA, Edgardo (Coord.). Historia de la educación en las provincias y territorios nacionales: (1885-1945). Buenos Aires: Galerna, 1993.

PINKASZ, Daniel; PITTELLI, Cecilia. Las reformas educativas en la Provincia de Buenos Aires (1934-1972) ¿Cambiar o conservar? In: PUIGGRÓS, Adriana; OSSANNA, Edgardo (Coord.) La educación en las provincias Argentinas (1945-1985). Buenos Aires: Galerna, 1997.

PLOTKIN, Mariano. Mañana es San Perón. Buenos Aires: Ariel, 1994.

PUIGGRÓS, Adriana (Dir.). Escuela, democracia y orden (1916-1943). Buenos Aires Galerna, 1992. Peronismo: cultura política y educación (1945-1955). Buenos Aires: Galerna, 1993. 
PUIGGRÓS, Adriana; OSSANNA Edgardo (Coord.). Historia de la educación en las provincias y territorios nacionales: (1885-1945). Buenos Aires: Galerna, 1997.

REIN, Raanan. Peronismo, populismo y política. Buenos Aires: Belgrano, 1998.

REPÚBLICA ARGENTINA. Ministerio de educación. Dirección General de Enseñanza Primaria. Educación Común en la capital, territorios y provincias. Talleres gráficos de Consejo Nacional de Educación, 1946.

SECRETARIA TÉCNICA. IV Censo General de la Nación. Buenos Aires, 1947. t. 1, v. 1.

SOMOZA RODRÍGUEZ, Miguel. Educación y política en la Argentina (1946-1955). Buenos Aires: Miño y Dávila, 2006.

TEDESCO, Juan Carlos. Educación y sociedad en la Argentina (1880-1945). Buenos Aires: Solar, 1986.

TORRE, Juan C.; PASTORIZA, Elisa. La democratización del bienestar en los años peronistas. In: TORRE, Juan C. (Comp.). Los años Peronistas: nueva historia Argentina, Buenos Aires, Sudamericana, 2002.t. 8.

WALTER, Richard. La provincia de Buenos Aires en la política Argentina 1912-1943. Buenos Aires: Emecé, 1987.

Recebido em 19/02/2012

Aprovado em 30/05/2012 